\section{The periodontal-systemic connection: a State of the Science symposium}

\author{
Richard Niederman ${ }^{1}$ and Raul Garcia ${ }^{2}$ \\ ${ }^{1}$ Forsyth Institute, Boston, Massachusetts, USA; \\ and ${ }^{2}$ Boston University, Goldman School of \\ Dental Medicine, Boston, Massachusetts, USA
}

Evidence-Based Dentistry (2002) 3, 17-19. DOI: 10.1038/sj/ ebd/6400088

The April 2001 conference sponsored by the American Academy of Periodontology and National Institute of Dental and Craniofacial Research focused on the relationship of periodontal disease to:

- Cardiovascular diseases;

- Respiratory diseases;

- Diabetes mellitus;

- Adverse pregnancy outcomes;

- Osteoporosis.

Except in the case of osteoporosis, the conceptual paradigm was the same (Figure 1). As host responses to infections are similar in periodontal disease and systemic infections, it is hypothesised that periodontal infections may generate harmful systemic effects.

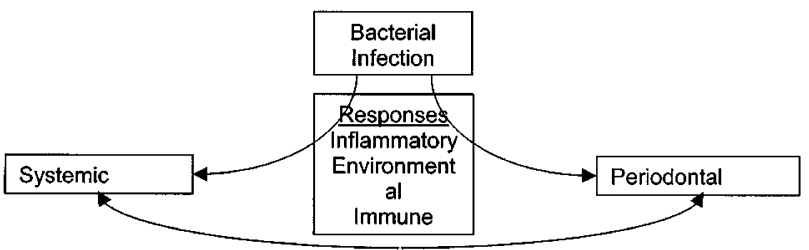

Figure 1 Bacterial infections stimulate a variety of direct and indirect host responses. As local bacterial infections and the host responses they generate can have significant detrimental systemic effects, periodontal infections therefore might have such harmful systemic effects. The converse hypothesis may also be true - that systemic disease can have a detrimental effect on the periodontium.

For osteoporosis specifically, it is hypothesised that systemic osteopenia is a risk factor for alveolar bone loss and thus for periodontal disease, ie, the systemic condition affects the oral condition.

The best reported evidence that supports the association between periodontal and systemic disease primarily comes from observational studies. There are, to date, no large randomised controlled trails demonstrating causality. Thus, a major caveat of reported work is whether the observed associations could represent residual confounding by, for example, tobacco smoking. In the conference poster session, three posters were of particular interest regarding the profound confounding effect of smoking, as follows.

\section{Concurrence of periodontitis and cancer}

\author{
P Hujoel et al., University of Washington, Seattle, USA
}

Hujoel et al. used the first National Health and Nutrition Examination Survey (NHANES-I), data set to examine the non-specificity of the periodontitis-systemic disease relationship by assessing the association of periodontitis with cancer. They tested the hypothesis that periodontal disease is a risk factor for cancer.

Table 1 Risk for periodontal disease causing lung cancer

\begin{tabular}{|c|c|c|c|}
\hline Cancer type & $\begin{array}{c}\text { Adjusted } \\
\text { odds ratio }\end{array}$ & $\begin{array}{c}95 \% \text { Confidence } \\
\text { interval }\end{array}$ & Controlled for: \\
\hline Any cancer & 1.55 & 1.25 to 1.92 & Age, gender \\
\hline Lung cancer & 1.94 & 1.16 to 3.26 & Age, gender \\
\hline Lung cancer & 1.73 & 1.02 to 2.97 & $\begin{array}{c}\text { All covariates including } \\
\text { smoking and socio-economic } \\
\text { status }\end{array}$ \\
\hline
\end{tabular}

The results (Table 1) suggest that periodontal disease is a risk factor for lung cancer. However, there is no biologic evidence for this association, although there is good evidence for the risk from smoking. These results imply that other reported associations between periodontitis and systemic health outcomes could also be explained by such residual confounders. Alternatively, periodontitis may be acting as a surrogate marker for unmeasured effects of smoking.

\section{Cigarette smoking, periodontal disease, and heart attack history}

J Hyman and DM Winn, National Institute of Dental and Craniofacial Research (NIDCR) and National Cancer Institute, Bethesda, Maryland, USA

Hyman and Winn used a cross-sectional study of National Health and Nutrition Examination Survey (NHANES) III data of about 6200 adults to examine the relationship of smoking and periodontitis with heart attacks. Two findings are notable. First, there was a statistically significant dose-response relationship between periodontal disease severity and heart attacks in smokers (Table 2). 
Table 2 Heart attacks in people who have periodontal disease and smoke

\begin{tabular}{lcc}
\hline $\begin{array}{l}\text { Mean attachment } \\
\text { level }\end{array}$ & $\begin{array}{c}\text { Adjusted } \\
\text { odds ratio }\end{array}$ & $\begin{array}{c}95 \% \text { Confidence } \\
\text { interval }\end{array}$ \\
\hline$>1-2 \mathrm{~mm}$ & 2.4 & $1.3-4.4$ \\
$>2-3 \mathrm{~mm}$ & 3.8 & $1.3-11.3$ \\
$>4 \mathrm{~mm}$ & 5.5 & $2.0-15.6$ \\
\hline
\end{tabular}

The second and more interesting finding was that there was no association between periodontal disease and heart attacks in non-smokers. The authors concluded that cigarette smoking is a necessary cofactor in the previously identified periodontal disease-heart disease relationships.

\section{Inadequate adjustment for smoking in periodontitis-systemic disease associations}

CF Spiekerman et al., University of Washington, Seattle, Washington, USA

'In silico' smoking experiments of Spiekerman and colleagues support the previous two sets of findings. Using published data on self-reported smoking and measured periodontal disease and serum cotinine levels (a direct measure of smoke inhalation) from 29 patients, they generated 1000 computer-simulated data sets. Using Cox proportional-hazard models, no periodontal diseasesystemic disease relationship was identified when the analysis was adjusted for serum cotinine levels. In contrast, when the analysis was adjusted for self-reported smoking, significant (false-positive) relationships were identified in $75 \%$ of the simulations. Thus, self-reported smoking, the method used in most studies to date, may not be adequate to adjust for the effects of smoking on the systemic health outcome.

\section{Cardiovascular disease}

J Beck, University of North Carolina, Chapel Hill, North Carolina, USA

Beck reviewed the initial cross-sectional and case-control studies that demonstrate a relationship between periodontal disease and cardiovascular disease. In contrast, more recent case-control and two longitudinal studies reported no statistical relationship. Several conceptual failings occurred in all of the studies:

- None of the studies were specifically designed to test the examined associations;
- All studies used a blunt index of periodontal disease (eg, previous attachment loss) rather than the infectious burden or the host response;

- All studies used blunt indices of outcome.

Beck also pointed out that the studies which found no association were those that used the crudest clinical measures. Given the potential impact this could have on health, he suggested that more specific methods be employed to further examine this relationship.

\section{Respiratory disease}

R Garcia, Boston University, Boston, Massachusetts, USA

A structured systematic review of the relationship between chronic obstructive pulmonary disease (COPD) and periodontal disease was presented by Garcia. The evidence that is available from secondary analyses of observational cross sectional and longitudinal studies indicates that poorer periodontal health status is associated with increased likelihood or risk of COPD. The risk ranges from 1.8 to 4.5. However, the role of periodontitis as a predictor of COPD appears to be limited to people who smoke (Table $3)$.

Table 3 COPD in subjects with periodontal disease

\begin{tabular}{lcc}
\hline Smoking status & Relative risk & 95\%Confidence interval \\
\hline All subjects & 1.54 & $1.26-1.90$ \\
Current smokers & 1.63 & $1.20-2.21$ \\
Never smoked & 1.19 & $0.71-1.98$ \\
\hline
\end{tabular}

The evidence for the association of oral infection and aspiration pneumonia is stronger and has greater biological plausibility, but the only reported clinical trials addressing this question each have important limitations.

\section{Diabetes}

G Taylor, University of Michigan, Ann Arbor, Michigan, USA; $S$ Grossi, State University of New York, Buffalo, New York, USA

Taylor presented a structured systematic review that suggests a bi-directional relationship between diabetes and periodontal disease. Of the studies identified, the vast majority of cross-sectional studies (43 out of 47) and all of the cohort studies (of which there were 
seven) provided consistent evidence of greater prevalence, severity, extent or progression of periodontal disease in diabetic patients.

Meanwhile, S Grossi reported on her randomised controlled trials using mechanical therapy and antibiotics. In these studies, the elimination of Propionibacterium gingivalis infection resulted in a concomitant reduction in glycated haemoglobin. This is in marked contrast to previous studies of mechanical periodontal therapy that demonstrated no effect on diabetes.

Both presentations support a direct relationship between periodontal disease and diabetes.

\section{Adverse pregnancy outcomes}

S Offenbacher et al., University of North Carolina, Chapel Hill, North Carolina, USA; M Jeffcoat, University of Alabama, Birmingham, Alabama, USA

Offenbacher reported on his group's prospective cohort study of 357 patients, examining the relationship between periodontal infection and abnormal pregnancy outcomes. Using multivariate models, the data indicate that there is an increased risk of adverse pregnancy outcomes in women who have periodontal disease (Table 4).

Table 4 Relationship between periodontal disease and adverse pregnancy outcomes

\begin{tabular}{lllll}
\hline & \multicolumn{2}{c}{ Prematurity } & $\begin{array}{c}\text { Low birth } \\
\text { weight } \\
<2500 \mathrm{~g}\end{array}$ & $\begin{array}{c}\text { Pre- } \\
\text { eclampsia }\end{array}$ \\
\cline { 2 - 4 } & $<37$ weeks & $<34$ weeks & $2.6(0.01)$ & $6.3(0.03)$ \\
\hline $\begin{array}{l}\text { Adjusted odds } \\
\text { ratio }(P)\end{array}$ & $1.9(0.03)$ & $3.0(0.03)$ & 2.6 & \\
\hline
\end{tabular}

M. Jeffcoat reported on her group's prospective study of 1300 pregnant women. In this group, the risk of preterm birth increased from three- to seven-fold with the severity of periodontal disease. Furthermore, the greatest risk of the earliest preterm birth ( $<32$ weeks) occurred in the women who had the most severe periodontal disease.

While supporting the reining hypothesis, these correlation studies await intervention studies that validate causality.

\section{Osteoporosis}

E Krall, Boston University, Boston, Massachusetts, USA

Distinct from the other periodontal-systemic associations, the proposed causal direction here is that systemic osteopenia is a risk factor for periodontal disease, via effects on alveolar bone support. It is important to note that there is no suggestion that the relationship works in the other direction, ie, periodontal infection is not a risk factor for systemic osteopenia.

A systematic review was presented by Krall, who described the effects on tooth retention and periodontal health status of various interventions intended to treat or prevent osteoporosis. The best evidence currently available on this relationship and on the effects of such interventions comes from observational studies of longitudinal cohorts (for example, the Framingham Study, VA Dental Longitudinal Study, Nurses' Health Study, Leisure World) and from secondary analyses of clinical trial data (eg, STOP-IT, Women's Health Initiative). The findings indicate that interventions which have a beneficial effect on systemic bone loss are also associated with lower risk or likelihood of tooth loss and alveolar bone loss.

To date, no results from clinical trials in this area have been published that used either tooth loss or periodontal health status as the primary outcome measures.

\section{Summary}

Using Bradford-Hill's criteria for demonstration of causality and the Centre for Evidence-based Medicine's categories for levels of evidence for demonstrating causality, there is clear biological plausibility for the hypothesised relationships. There are also consistent findings from multiple sources to support those relationships. The levels of evidence, however, range from a host of cross-sectional studies which do not demonstrate causality but are hypothesis-generating, to a number of case series and retrospective case-control trials, to two cohort trials (adverse pregnancy outcomes) and one randomised controlled trial (diabetes). The clear take-home message is the need for, first, higher levels of clinical evidence that demonstrate or refute the causal hypothesis and, second, analysis and controls to account for the contribution of smoking. 